\title{
Reactividad fisiológica periférica y actividad cerebral en las fobias específicas
}

\author{
Juan Pedro Sánchez Navarro, José María Martínez Selva \\ Departamento de Anatomía Humana y Psicobiología. Universidad de Murcia
}

Disponible online 22 diciembre de 2009

\begin{abstract}
Las fobias específicas son miedos exagerados e irracionales a estímulos concretos acompañados de reacciones fisiológicas y conductuales de lucha o huida. A nivel periférico, se produce un aumento de la reactividad vegetativa y somática, como muestran distintos índices fisiológicos (frecuencia cardiaca, respuesta electrodérmica), y una potenciación de los reflejos defensivos (respuesta cardiaca de defensa o parpadeo reflejo de sobresalto). A nivel de sistema nervioso central, se ha descrito una red de estructuras cerebrales relacionadas con el procesamiento y la respuesta al estímulo fóbico. Esta red está compuesta por la amígdala, la corteza órbitofrontal, la corteza cingulada anterior y la ínsula anterior. La respuesta fóbica se caracteriza por un aumento de actividad en estas regiones, que se puede asociar con los cambios vegetativos y somáticos, la percepción subjetiva de miedo intenso y las conductas de evitación del estímulo fóbico.
\end{abstract}

Palabras clave: Fobias específicas, actividad vegetativa, reflejos defensivos, amígdala, corteza prefrontal, ínsula

Specific phobias are exaggerated and irrational fears caused by specific stimuli. These anxiety disorders can appear together with physiological reactions and fight or flight responses. At a peripheral level the phobic response is featured by an increase in somatic and autonomic reactivity as shown by different physiological indices (heart rate, electrodermal activity) and a potentiation of defensive reflexes, such as the cardiac defense response and the blink reflex. At a central level it has been described a network of brain structures that are involved both in the processing of the phobic stimulus and in the reaction that it provokes. This brain network is composed by the amygdala, the orbitofrontal and cingulate cortices and the anterior insula. An increase in the activity of these brain regions occurs during the phobic reaction that can be associated with the somatic and autonomic changes, the subjective experience of intense fear and the avoidance behavior elicited by the phobic stimulus.

Keywords: Specific phobia, autonomic activity, defensive reflexes, amygdala, prefrontal cortex, insula.

\footnotetext{
Este trabajo es resultado del proyecto de investigación 08839/PHCS/08 financiado con cargo al Programa de Generación del Conocimiento Científico de Excelencia de la Fundación Séneca-Agencia de Ciencia y Tecnología de la Región de Murcia en el marco del II PCTRM 2007-10.

Correspondencia: Juan Pedro Sánchez Navarro. Departamento de Anatomía Humana y Psicobiología. Facultad de Psicología. Campus de Espinardo. Universidad de Murcia. 30100 Murcia (España).E-mail: jpedro@um.es
} 
La detección de cambios repentinos e inesperados en el ambiente, así como centrar la atención y reaccionar ante ellos, son respuestas críticas para la supervivencia. Además, resulta fundamental ignorar los estímulos irrelevantes para enfrentarse a otros más importantes. En función de la naturaleza y la relevancia del estímulo, el organismo puede reaccionar con una respuesta de orientación o con una respuesta de defensa (Sokolov, 1963; ver Graham, 1979 para otras respuestas, como la de sobresalto). La respuesta de defensa suele estar provocada por estímulos muy intensos o potencialmente dañinos y posee una función protectora frente a ellos, al atenuar los inputs sensoriales y facilitar las reacciones de retirada o lucha. En estas situaciones, los humanos reaccionan con percepción subjetiva de miedo, acompañada de un abanico de respuestas defensivas, como la inmovilización y la lucha o la huida (Lang, Davis y Öhman, 2000; Vila, Guerra, Muñoz, Vico, Viedma-Del Jesús, Delgado et al., 2007).

De acuerdo con la adaptación del modelo de Fanselow (1994) llevada a cabo por Lang, Bradley y Cuthbert (1997), la reacción del organismo a una amenaza se compone de una secuencia de respuestas. La fase inicial se caracteriza por reacciones atencionales o de orientación focalizadas en el estímulo amenazante. Esta fase se relaciona con la detección y el análisis del estímulo potencialmente peligroso mediante una actitud vigilante (Vila et al., 2007), y se correspondería con la reacción de defensa preparatoria propuesta por Masterson y Crawford (1982). En esta situación se produce un aumento tanto de la actividad vegetativa simpática como parasimpática, aunque con un predominio de esta última. La fase final (fase 3 de Fanselow) se caracteriza por el desencadenamiento de acciones dirigidas a la defensa activa del sujeto, y se correspondería con la reacción de alarma propuesta por Masterson y Crawford (1982), en la que las respuestas de orientación pasan a convertirse en acciones de defensa (Graham, 1979; Sokolov, 1963). De este modo se produce un aumento de la actividad vegetativa simpática, lo que se traduce, entre otros cambios, en un aumento de la conductancia de la piel y de la frecuencia cardiaca.

Siguiendo este modelo, el miedo es una emoción de tipo defensivo provocada por la aparición (real o simbólica) de un estímulo que supone una amenaza, real o imaginada, para el sujeto. El miedo, por tanto, tiene la función de proteger al organismo de peligros potenciales para su supervivencia. Como en cualquier emoción, en el miedo se puede distinguir una actividad músculo-esquelética observable (conducta) relacionada con la evitación, huida o enfrentamiento con el objeto de miedo, cambios neuroendocrinos a nivel central y periférico y una fuerte percepción subjetiva de temor. Debido a su función adaptativa, estos componentes del miedo se han mantenido a lo largo de la evolución de los mamíferos. Sin embargo, en la actualidad, el ser humano puede mostrar reacciones defensivas intensas que no se ajustan a la presencia de una amenaza real para la supervivencia. La aparición de estas respuestas defensivas ante estímulos reales o simbólicos (p.e., una fotografía o la imaginación) no relacionados con un peligro potencial para el sujeto es lo que caracteriza a la fobia. Una fobia específica es un miedo concreto, irracional y persistente a un determinado estímulo (p.e., arañas o serpientes), de tal modo que la mera confrontación con el mismo produce una reacción subjetiva de ansiedad severa y aguda (Fyer, 1998). Por lo tanto la fobia es un miedo intenso provocado por un estímulo que no justifica la aparición de dicho miedo desde la perspectiva de la supervivencia del sujeto, y que se acompaña de una sobreactivación del sistema de defensa del organismo, dando lugar a una fuerte activación vegetativa simpática, y de un aumento de la actividad de varias regiones cerebrales. Esta reactividad fisiológica y cerebral aparece incluso cuando las personas no perciben de forma consciente los estímulos fóbicos, lo que indica que estas reacciones defensivas surgen rápidamente por medio de mecanismos de procesamiento preatencional.

Dado que los cambios fisiológicos y de actividad cerebral son componentes constitutivos de cualquier emoción, y dado el importante papel que juegan en el desencadenamiento de las mismas, a lo largo de este trabajo nos centraremos en las reacciones fisiológicas que se producen en el sujeto fóbico cuando se expone al estímulo de su fobia, así como en los cambios que se producen en su actividad cerebral.

Reactividad fisiológica provocada por la estimulación fóbica

La principal reacción fisiológica que se observa en los sujetos fóbicos cuando se les confronta con estímulos relacionados con su fobia (p.e., arañas o serpientes) es un aumento de la actividad vegetativa simpática, con efectos sobre diferentes órganos y glándulas del organismo, que da lugar a diferentes respuestas.

En la vida real, los estímulos potencialmente peligrosos son complejos y pueden suponer una amenaza efectiva para la integridad del organismo, en comparación con los estímulos empleados en el laboratorio. No obstante, algunos autores han encontrado que las imágenes, como fotografías, son capaces de provocar emociones similares debido a que poseen un significado representativo o simbólico para el sujeto equivalente a las propiedades del estímulo real. De este modo, pueden activar las representaciones cognitivas relacionadas con las respuestas emocionales y dar lugar a reacciones fisiológicas y conductuales similares a las provocadas por el estímulo real (Lang, 1995; Lang et al., 1997; Lang, Greenwald, Bradley y Hamm, 1993). Por lo tanto, la mayor parte de los datos que se tienen en la actualidad sobre la reacción provocada por la estimulación fóbica en sujetos fóbicos proceden de estudios que han empleado imágenes como estímulos más que de aquéllos que se valen de una exposición real a la estimulación fóbica (si bien es cierto que en algunos casos de fobias específicas resulta imposible realizar en el laboratorio). 
Respuestas periféricas provocadas por estímulos fóbicos

a) Actividad cardiovascular

Uno de los sistemas más estudiados en relación a las respuestas defensivas ha sido el cardiovascular, y en particular, los cambios en el ritmo cardiaco. Brevemente, el sistema cardiovascular se puede dividir en el sistema circulatorio (vasos sanguíneos) y el músculo cardiaco (corazón), así como el conjunto de mecanismos que hacen posible que todo el sistema opere acompasadamente en condiciones de ausencia de daños estructurales o funcionales. Mientras que los vasos sanguíneos poseen una inervación exclusiva de la rama simpática del sistema nervioso vegetativo (cuyo aumento de actividad produciría vasoconstricción), el corazón presenta una doble inervación: vagal o parasimpática y simpática. Clásicamente se pensaba que ambas ramas vegetativas actuaban de forma recíproca, es decir, que el aumento de una de ellas se acompañaba de una disminución de la otra. Sin embargo, en la actualidad sabemos que ambas ramas pueden actuar, además de este modo, también de forma conjunta e incluso independientemente la una de la otra (Berntson, Cacioppo y Quigley, 1996; Berntson, Cacioppo, Quigley y Fabro, 1994). En general, el aumento de la actividad parasimpática sobre el corazón provoca una disminución de la frecuencia cardiaca, mientras que el aumento de la actividad simpática provoca tanto un aumento de la fuerza de contracción como aceleración cardiaca (Brownley, Hurwitz y Schneiderman, 2000; Papillo y Shapiro, 1990). Un aumento de la frecuencia cardiaca puede deberse a una disminución de la actividad parasimpática, a un aumento de la actividad simpática o a una interacción recíproca de la actividad de ambas ramas del sistema nervioso vegetativo sobre el nódulo sinoatrial. Del mismo modo, una desaceleración cardiaca puede estar provocada por un aumento de la actividad parasimpática, una disminución de la actividad simpática o por la interacción de ambas ramas vegetativas.

En general, los estudios que han utilizado imágenes para provocar emociones han encontrado un patrón bradicárdico (desacelerativo) que se acentúa en aquellas imágenes consideradas por los sujetos como más interesantes (esto es, las de afecto positivo y negativo, en comparación con las imágenes neutras). El patrón cardiaco es más complejo que una simple desaceleración y presenta la forma de una onda trifásica, compuesta por una breve desaceleración inicial, seguida por un incremento de la frecuencia cardiaca (aunque continúa bajo la línea base), seguido, finalmente, por una segunda desaceleración más marcada (Cook y Turpin, 1997; Lang et al., 1993, 1997). La frecuencia cardiaca promedio de esta secuencia es desacelerativa, lo que indica que es una respuesta relacionada con la atención. De acuerdo con la interpretación de Lang y colaboradores (1997), este patrón cardiaco se concibe como una cadena de reacciones que comienzan con una respuesta atencional de origen vagal. Esta reacción parasimpática se relaciona tanto con la conducta de inmovilización (freezing), que caracteriza la fase vigilante o preparatoria de la respuesta a una amenaza potencial, y que es similar a la bradicardia relacionada con el miedo en animales (Bradley y Lang, 2007), como con las características de una situación que no requiere una respuesta motora activa.

La respuesta defensiva, en forma de aceleración cardiaca, no aparece en el momento en el que se está produciendo la entrada de información, sino más adelante, cuando el sujeto percibe (consciente o inconscientemente) el estímulo como amenazante. Este componente acelerativo de la respuesta a imágenes aversivas tiene la función de preparar al organismo para una respuesta motora. Sin embargo, ocurre una excepción en sujetos fóbicos, quienes sólo presentan un patrón cardiaco acelerativo de corta latencia cuando se les muestra imágenes relacionadas con sus fobias (Cook, Davis, Hawk, Spence y Gautier, 1992; Hamm, Cuthbert, Globisch y Vaitl, 1997; Hare, 1973; Klorman, Weissberg y Wiesenfeld, 1977). En particular, se ha comprobado que los sujetos fóbicos, ante imágenes de sus fobias, presentan aceleración cardiaca, vasoconstricción cefálica y un aumento de la presión arterial, además de mostrar respuestas electrodérmicas de mayor magnitud y más resistentes a la habituación así como mayores respuestas de sobresalto (Globisch, Hamm, Esteves y Öhman, 1999; Hamm et al., 1997; Hare, 1973). Además, esta respuesta cardiaca a estímulos fóbicos puede condicionarse a un estímulo neutro. Cuando un estímulo acústico neutro, como un tono auditivo, se presenta antes de la imagen fóbica, los sujetos fóbicos aprenden a anticipar la aparición posterior de la imagen y muestran una aceleración cardiaca, mientras que los no fóbicos no muestran esta respuesta acelerativa (Hare y Blevings, 1975). La función de esta respuesta anticipadora podría considerarse una preparación para rechazar o atenuar el estímulo amenazante que anticipa. Pero no sólo las imágenes fóbicas provocan este patrón, sino que la sola imaginación de los estímulos fóbicos produce una mayor aceleración cardiaca que cuando se imaginan otros contenidos (Cook, Hawk, Davis y Stevenson, 1991). Estos datos parecen apoyar la hipótesis de la existencia de una red cerebral para el miedo que puede activarse de forma rápida (Globisch et al., 1999). Dicha red estaría asociada con la activación de la rama simpática del sistema nervioso vegetativo y relacionada con el desencadenamiento de la respuesta de defensa.

Sin embargo, entre las fobias específicas se encuentra una excepción a este patrón cardiaco, el caso de las fobias a la sangre. Ante imágenes relacionadas con su fobia, estos sujetos parecen mostrar una reactividad simpática inicial y pasajera, seguida de una fuerte respuesta vagal. Este patrón ha sido mostrado claramente en estudios que han presentado imágenes fóbicas a estos sujetos, obteniendo una marcada desaceleración cardiaca (Hamm et al., 1997; Klorman et al., 1977). El único aumento en la frecuencia cardiaca parece producirse en el primer ensayo (primera imagen fóbica) de una secuencia de varias imágenes. La naturaleza de este tipo de reactividad en los sujetos con fobia a la sangre no se conoce bien, aunque la investigación reciente está tratando de caracterizar este patrón car- 
diovascular atípico así como su naturaleza fisiológica y cerebral (Buodo, Peyk, Junghöher, Palomba y Rockstroh, 2007; Sarlo, Buodo, Munafló, Stegagno y Palomba, 2008; Sarlo, Palomba, Angrilli y Stegagno, 2002).

\section{b) Actividad electrodérmica}

La actividad electrodérmica es un índice vegetativo que tiene su origen en las glándulas sudoríparas ecrinas, cuya inervación es exclusivamente simpática. Una amplia variedad de estudios ha mostrado su relación lineal con el arousal provocado por la estimulación, apareciendo una respuesta mayor ante los estímulos más activadores (Lang et al., 1993; SánchezNavarro, Martínez-Selva, Torrente y Román, 2008). Es decir, con independencia del tono afectivo de las imágenes empleadas, aquéllas que provocan una mayor activación en el sujeto (p.e., eróticas, mutilaciones, etc.) suscitan una respuesta más intensa que las que no provocan tanta activación (p.e., una puesta de sol o un plato).

Ante imágenes relacionadas con sus fobias (p.e., arañas o serpientes) los sujetos fóbicos muestran respuestas de mayor magnitud que ante otros contenidos desagradables, y mayor también que las respuestas dadas por sujetos no fóbicos (Hamm et al., 1997; Wendt, Lotze, Weike, Hosten y Hamm, 2008). Además, estas respuestas al estímulo fóbico se mantienen en el tiempo, esto es, muestran menos habituación ante la aparición sucesiva de estímulos fóbicos, a diferencia de lo que ocurre con otros contenidos y de lo que sucede en sujetos no fóbicos (Wendt et al., 2008).

Este aumento de la respuesta electrodérmica también se ha observado ante imágenes presentadas fuera de la percepción consciente del sujeto (subliminalmente). Los estudios de Öhman han mostrado que la percepción no consciente de imágenes fóbicas produce una mayor respuesta electrodérmica que otros tipos de imágenes (Öhman y Soares, 1994). Este resultado apoya la hipótesis de Öhman acerca de la existencia de mecanismos de procesamiento preatencionales o automáticos, es decir, que se producen fuera de la conciencia del sujeto detectando rápidamente los estímulos de miedo.

\section{Potenciación de los reflejos defensivos}

a) Respuesta de sobresalto

La respuesta de sobresalto es una reacción de todo el organismo que se produce por la aparición repentina de un estímulo de alta intensidad. En el ser humano, una de las primeras manifestaciones de esta respuesta defensiva es el parpadeo reflejo. Se trata de un reflejo muy rápido, con una latencia entre 21 y $120 \mathrm{~ms}$. aproximadamente. Distintos estudios han mostrado que tanto su magnitud como su latencia se encuentran moduladas por el valor emocional del contexto en el que se produce (Lang, 1995). Así, se produce una potenciación de esta respuesta si se desencadena mientras el sujeto observa una imagen de contenido desagradable, y una atenuación del mismo si se provoca durante la visión de imágenes agradables. A este efecto se le conoce como modulación emocional del parpadeo reflejo de sobresalto. Lang (1995; Lang et al., 1997) ha propuesto el modelo de priming motivacional, según el cual cuando los sujetos se encuentran en un estado motivacional de tipo defensivo o aversivo, todas las representaciones y programas de acción relacionadas con dicho estado motivacional se encontrarían potenciadas, mientras que las representaciones y programas de acción del estado motivacional apetitivo se encontrarían atenuadas. Así, cuando el sujeto se encuentra observando imágenes de contenido desagradable, su estado motivacional dominante sería el defensivo. Si en ese momento se presenta un estímulo acústico intenso e inesperado, se produce un parpadeo reflejo más intenso dado que todos los programas de acción relacionados con el estado motivacional aversivo están potenciados, y el reflejo de sobresalto pertenece a ese repertorio al tratarse de un reflejo defensivo. Sin embargo, si el sujeto se encuentra en un estado apetitivo, la aparición de un sonido intenso e inesperado produce una respuesta de sobresalto menor, dado que este reflejo no forma parte del repertorio apetitivo y, por lo tanto, no se encuentra potenciado sino atenuado.

Esta respuesta también parece encontrarse potenciada cuando se provoca en sujetos fóbicos. La respuesta de sobresalto es mayor cuando se provoca ante imágenes relacionadas con sus fobias que ante otros tipos de imágenes, y también es mayor que en sujetos no fóbicos (Hamm et al., 1997; Pissiota, Frans, Michelgard, Appel, Langström, Flaten et al., 2003; Wendt et al., 2008). Además, se ha visto que esta potenciación de la respuesta ante imágenes fóbicas surge antes que frente a otros tipos de imágenes, lo que corrobora que la respuesta de miedo puede activarse muy rápidamente (Globisch et al., 1999). Pero no sólo su potenciación es más rápida, sino que también opera de forma inconsciente, ya que los fóbicos muestran una potenciación de esta respuesta ante las imágenes fóbicas, presentadas de forma subliminal, en comparación con otros contenidos afectivos no fóbicos (Ruiz-Padial y Vila, 2007).

Por último, también se ha observado que la respuesta de sobresalto es un buen indicador que permite distinguir entre las fobias específicas, ya que, en el caso de fobias de carácter filogenético (p.e., serpientes o arañas) se aprecia una potenciación de esta respuesta ante las imágenes fóbicas en comparación con otros tipos de imágenes desagradables, mientras que en el caso de miedos ontogenéticos (p.e., miedo a volar) no se aprecia dicha modulación (Mühlberger, Wiedemann, Hermann y Pauli, 2006).

\section{b) Respuesta cardiaca de defensa}

Se pueden provocar respuestas cardiacas defensivas en humanos empleando diferentes tipos de estímulos, como un ruido blanco o un shock eléctrico. La respuesta cardiaca de defensa resultante es un patrón complejo de fluctuaciones del latido cardiaco. Ante un estímulo acústico intenso y abrupto, este patrón defensivo tiene una duración aproximada de 80 segundos, y está compuesto por dos componentes acelerativos 
y dos desacelerativos que aparecen en orden alterno (SánchezNavarro y Martínez-Selva, 2009; Sánchez-Navarro, MartínezSelva y Román, 2006; Vila et al., 2003, 2007). Se ha comprobado una influencia diferencial de ambas ramas del sistema nervioso vegetativo sobre cada uno de estos componentes, tanto en animales como en humanos (Quigley y Berntson, 1990; Reyes del Paso, Vila y García, 1994). Los datos obtenidos en humanos muestran una mayor influencia parasimpática en los dos primeros componentes de la respuesta (primera aceleración y primera desaceleración), mientras que los dos segundos componentes (segunda aceleración y segunda desaceleración) se encontrarían mediados por una interacción recíproca de las ramas simpática y parasimpática, aunque con predominio simpático (Fernández y Vila, 1989; Reyes del Paso, Godoy y Vila, 1993; Vila et al., 2003, 2007). Recientemente se ha realizado una reinterpretación del significado de los componentes de esta respuesta (Vila et al., 2007), relacionándolos tanto con procesos atencionales como motivacionales (Ramírez, Sánchez, Fernández, Lipp y Vila, 2005). Los dos primeros componentes (aceleración y desaceleración de corta latencia) estarían relacionados con la vigilancia, orientación y preparación de respuestas, es decir, con la interrupción de la actividad en curso y la focalización de la atención sobre la amenaza potencial, indicando una transición desde la atención a la acción (Vila et al., 2007). Los dos últimos componentes (aceleración y desaceleración de larga latencia) reflejarían una respuesta de defensa y estarían relacionados con conductas defensivas (de lucha o huida) para enfrentarse con la amenaza.

Esta respuesta defensiva ha sido estudiada en muestras de pacientes con fobias específicas. Partiendo del modelo de priming motivacional propuesto por Lang (1995; Lang et al., 1997), se ha observado un aumento de los componentes acelerativos de la respuesta cardiaca de defensa cuando ésta se provoca (mediante un ruido intenso) mientras que los sujetos fóbicos se encuentran viendo imágenes relacionadas con sus fobias (Sánchez, Ruiz-Padial, Pérez, Fernández, Cobos y Vila, 2002). En particular, el patrón de la respuesta cardiaca es diferente al observado cuando la respuesta se provoca en ausencia de una imagen (presentando solamente el estímulo acústico, como se ha descrito anteriormente), caracterizándose ahora por una única y mayor aceleración, en la que desaparece la primera desaceleración. Los autores han interpretado este cambio en el patrón cardiaco como un avance de fase de la reacción defensiva, es decir, un avance en el tiempo de la segunda aceleración cardiaca debido a que la respuesta a la amenaza (estímulo acústico) se encuentra facilitada por las imágenes fóbicas, lo que se traduciría en un adelanto de las acciones protectoras defensivas (Ruiz-Padial, Mata, Rodríguez, Fernández y Vila, 2005).

Pero no sólo la percepción de imágenes fóbicas provoca esta potenciación de la respuesta cardiaca de defensa, sino que la presentación subliminal de estos estímulos produce una respuesta similar en sujetos fóbicos, aunque de menor magnitud (Ruiz-Padial et al., 2005). Es decir, la percepción no consciente del objeto fóbico provoca una potenciación de la respuesta cardiaca de defensa, lo que es congruente con el modelo de Öhman según el cual los estímulos que provocan miedo se procesan de un modo preatencional, permitiendo su detección y respuesta rápidas.

Actividad cerebral provocada por la estimulación fóbica

La investigación reciente no solamente ha intentado caracterizar el procesamiento cognitivo de estímulos fóbicos y las respuestas motoras (conductuales) y vegetativas, sino que, gracias al avance de las técnicas electrofisiológicas y de neuroimagen, ha dado un salto cualitativo en la descripción de los mecanismos neuronales implicados en las fobias específicas y en la explicación de estos fenómenos patológicos. Desde la perspectiva de la neuroanatomía funcional clásica, se ha considerado a la amígdala como la principal estructura implicada tanto en el procesamiento como en la respuesta rápida a estímulos fóbicos (Sánchez-Navarro y Román, 2004). Sin embargo, otras estructuras cerebrales parecen encontrarse también implicadas, como la corteza prefrontal ventromedial (Carretié, Albert, LópezMartín y Tapia, 2009), la porción anterior de la circunvolución del cíngulo (Goossens, Sunaert, Peeters, Griez y Schruers, 2007a; Goossens, Schruers, Peeters, Griez y Sunaert, 2007b) y la ínsula anterior (Etkin y Wager, 2007).

Las respuestas emocionales dependen de dos sistemas motivacionales del cerebro (Bradley, 2000; Bradley y Lang, 2000; Lang et al., 1990, 1997), el sistema apetitivo, relacionado con conductas de aproximación (como la ingesta de comida o la copulación) y el sistema defensivo o aversivo, relacionado con conductas de evitación, retirada y defensa. Los estudios llevados a cabo tanto con animales como con humanos han mostrado que la amígdala cerebral es una estructura clave en el así llamado "sistema cerebral del miedo" (Sánchez-Navarro y Martínez-Selva, 2009; Sánchez-Navarro et al., 2006).

\section{Amígdala}

La amígdala desempeña un papel fundamental tanto en las respuestas emocionales como en las defensivas, particularmente en el procesamiento de, y en la respuesta a, estímulos amenazantes (Aggleton y Young, 2000). La amígdala está formada por un conjunto de núcleos situados en la cara medial anterior del lóbulo temporal, formando parte del sistema límbico. Esta estructura recibe información aferente de todas las áreas sensoriales de asociación, incluyendo las viscerales (Aggleton y Young, 2000; Ben-Ari, 1981; LeDoux, 1987), así como proyecciones neuronales de la corteza órbitofrontal, el sector CA1 del hipocampo, subiculum, hipotálamo, estructuras olfatorias, núcleo del tracto solitario y sustancia innominada (Ben-Ari, 1981; Emery y Amaral, 2000). Dada esta convergencia de proyecciones, la amígdala se ha constituido como una región clave para la formación de asociaciones entre estímulos y refuerzos (LeDoux, 2000; Murray, 2007; Rolls, 1986). 
Pero, además de esta ingente cantidad de proyecciones neuronales, la amígdala también recibe aferencias procedentes del tálamo (LeDoux, 1987, 1993). La información sensorial que llega a los núcleos talámicos se envía a regiones corticales para un análisis perceptivo complejo. Sin embargo, las características más simples de estos estímulos también se proyectan desde el tálamo hasta la amígdala. Se ha sugerido que estas características simples de los estímulos activan los circuitos emocionales de la amígdala, preparando a esta estructura para recibir la información más compleja del estímulo procesada en la corteza cerebral (LeDoux, 1989). A su vez, la amígdala envía proyecciones a una gran cantidad de estructuras corticales y subcorticales, modulando el procesamiento de los estímulos y las respuestas fisiológicas y conductuales provocadas por tales estímulos. Así, a través de sus proyecciones eferentes hacia el hipotálamo lateral, sustancia gris periacueductal, núcleo ambiguo, núcleo del tracto solitario, núcleo del lecho de la estría terminal, núcleo motor dorsal del vago, médula ventrolateral rostral y núcleo paragigantocular, la amígdala estaría implicada en la modulación de las respuestas vegetativas y conductuales que aparecen en emociones tales como el miedo (Berntson y Cacioppo, 2007; Berntson, Quigley y Lozano, 2007; LeDoux, 1987; LeDoux, Iwata, Cicchetti y Reis, 1988). De igual modo, a través de sus proyecciones eferentes, pone en marcha los sistemas activadores del cerebro, favoreciendo el análisis de los estímulos potencialmente amenazantes, aumentando el nivel de vigilancia y la optimización en el procesamiento de la información, lo que permite un análisis más preciso de los estímulos, y realizando una preparación de las respuestas más apropiadas (Aggleton y Young, 2000; Davis, 1997; LeDoux, 2000). Por lo tanto, la amígdala es una estructura clave para realizar un procesamiento rápido de los estímulos amenazantes, o con más relevancia, y para la preparación o el desencadenamiento de una respuesta rápida ante ellos.

Los estudios de neuroimagen funcional realizados en humanos han corroborado esta implicación de la amígdala en el procesamiento de estímulos emocionales en general, y en el de estímulos que provocan miedo en particular (LaBar, LeDoux, Spencer y Phelps, 1995; Lane, Reiman, Bradley, Lang, Ahern, Davidson et al., 1997; Morris, Frith, Perrett, Rowland, Young, Calder et al., 1996). Ante la exposición a estímulos visuales emocionales, las imágenes clasificadas como negativas provocan una mayor activación amigdalina (Canli, Sivers, Whitfield, Gotlib y Gabrieli, 2002; Critchley, Rotshtein, Nagai, O’Doherty, Mathias y Dolan, 2005; Das, Kemp, Liddell, Brown, Olivieri, Peduto et al., 2005). De igual modo, las palabras catalogadas como desagradables también provocan una mayor activación de esta estructura que las palabras neutras (Tabert, Borod, Tang, Lange, Wei, Jonson et al., 2001). Por lo tanto, algunos autores sugieren que esta activación diferencial de la amígdala a estímulos desagradables se debe a que es necesaria para responder de un modo estereotipado y universal a estímulos amenazantes o que señalan peligro, sin la necesidad de un proce- samiento cognitivo complejo, proporcionando una preparación rápida para la acción (Paradiso, Jonson, Andreasen, O'Leary, Watkins, Ponto et al., 1999). Sin embargo, se debe mencionar que algunos estudios también han mostrado que la amígdala podría estar relacionada con la relevancia de los estímulos con independencia de su valencia afectiva (Phan, Pager, Taylor y Liberzon, 2002), ya que aparece una mayor actividad de esta estructura ante estímulos positivos y negativos en comparación con estímulos neutros (Hamann, Ely, Hoffman y Kilts, 2002; Sabatinelli, Bradley, Fitzsimmons y Lang, 2005).

La amígdala parece desempeñar una función central tanto en el procesamiento de estímulos fóbicos como en la respuesta defensiva de miedo provocada por éstos (LeDoux, 1998). En el caso de las fobias a animales (p.e., arañas o serpientes) se aprecia una mayor actividad amigdalina cuando los sujetos fóbicos observan imágenes relacionadas con su miedo fóbico que cuando se les presentan imágenes con otros contenidos desagradables (Sabatinelli et al., 2005; Ahs, Pissiota, Michelgard, Frans, Furmark, Appel et al., 2009). Esta actividad es mayor que la que aparece en sujetos no fóbicos y parece ser independiente de la percepción consciente del estímulo fóbico, ya que se da aun cuando el sujeto no perciba de forma consciente el estímulo fóbico, como cuando se emplean procedimientos de enmascaramiento para la presentación subliminal de estímulos (Carlsson, Petersson, Lundqvist, Karlsson, Ingvar y Öhman, 2004; Dilger, Straube, Mentzel, Fitzek, Reichenbach, Hecht et al., 2003). En este sentido, se ha encontrado una mayor activación amigdalina tanto a las imágenes fóbicas como a las imágenes negativas no fóbicas cuando las imágenes se presentan de forma enmascarada, actividad que podría estar indicando una respuesta a estímulos que "podrían tener relevancia" para la supervivencia; sin embargo, cuando las imágenes se presentan rápidamente, pero no enmascaradas, la actividad amigdalina es mayor para los estímulos fóbicos que para los estímulos no fóbicos, lo que se interpreta como una respuesta a los estímulos que "tienen relevancia" para la supervivencia del sujeto (Carlsson et al., 2004). Además, cuando el estímulo fóbico es percibido de forma consciente, también se aprecia un aumento de actividad en todo un conjunto de estructuras cerebrales, incluyendo la ínsula anterior, que se ha relacionado con la representación del estado corporal interno, la corteza órbitofrontal y la corteza cingulada anterior (Carlsson et al., 2004). Resultados similares, incluyendo toda esta red de estructuras cerebrales, han sido hallados en otros estudios que han manipulado la atención del sujeto hacia el estímulo fóbico. Cuando la atención del sujeto se focaliza en la imagen fóbica, se produce un aumento de actividad en toda esta red de estructuras cerebrales, mientras que cuando la atención del sujeto no se dirige hacia el estímulo fóbico se aprecia un aumento en la actividad de la amígdala, lo que refuerza la hipótesis de que esta estructura realiza un procesamiento automático de los estímulos relevantes para la supervivencia, es decir, de los estímulos amenazantes o que señalan amenaza, en este caso estímulos fóbicos (Straube, Mentzel y Miltner, 2006b). 
Además de un mayor nivel de activación, también se ha observado una respuesta amigdalina más rápida en fóbicos expuestos a imágenes relacionadas con sus objetos fóbicos que en sujetos no fóbicos (Larson, Schaefer, Siegle, Jackson, Anderle y Davidson, 2006). La amígdala, por lo tanto, está particularmente implicada en el procesamiento automático de la amenaza, desempeñando una función especial en la detección y respuesta al estímulo fóbico, más que en el mantenimiento en el tiempo de la respuesta de miedo (Straube et al., 2006b). Esto último es de vital importancia a la hora de evaluar la respuesta de la amígdala a la estimulación fóbica, ya que algunos trabajos no han encontrado dicha actividad debido a que han utilizado tiempos de exposición demasiado largos, como Wendt et al. (2008), mientras que la respuesta amigdalina es rápida y de corta duración.

Los datos procedentes de pacientes fóbicos sometidos a tratamiento cognitivo-conductual avalan la participación de estas estructuras en el miedo fóbico (Goossens et al., 2007a; Straube, Glauer, Dilger, Mentzel y Miltner, 2006a). Estos estudios parten de la hipótesis de que la actividad observada en la amígdala, así como en otras regiones, como la ínsula anterior o la corteza cingulada anterior, cuando los sujetos fóbicos son expuestos a imágenes relacionadas con sus fobias, disminuirá y será similar a la observada en sujetos no fóbicos una vez que se haya completado el tratamiento cognitivo-conductual. Empleando una terapia de exposición conductual, Goossens et al. (2007a) encuentran que antes de la terapia los fóbicos expuestos al objeto de sus fobias (arañas o serpientes) mostraban una mayor activación en la amígdala, ínsula anterior y corteza cingulada anterior. Sin embargo, tras el tratamiento los pacientes mostraban una disminución de actividad en dichas regiones que era similar a la observada en los sujetos no fóbicos. Otros estudios han hallado resultados similares en la corteza cingulada anterior y en la ínsula (Straube et al., 2006a).

\section{Región prefrontal}

Otra región que se ha relacionado con la detección rápida de estímulos amenazantes es la región órbitofrontal. Se trata de una región polimodal que recibe proyecciones de todas las áreas sensoriales, así como de la amígdala, la corteza entorrinal y la corteza cingulada (Barbas, 2000). Envía proyecciones hacia la corteza temporal inferior, la corteza entorrinal, la corteza cingulada, el hipotálamo lateral, la amígdala, el área tegmental ventral, la cabeza del núcleo caudado y la corteza motora (Kemp y Powell, 1970; Nauta, 1964; vanHoesen, Pandya y Butters, 1975). Debido a su gran conectividad, esta región está relacionada con la integración de la información sensorial procedente de otras regiones cerebrales, la modificación de respuestas vegetativas y motoras y la modulación del procesamiento cognitivo (Morris y Dolan, 2004). La corteza órbitofrontal, particularmente la región ventromedial (constituida por el área 25 de Brodmann, porción inferior de las áreas 24 y 32 y sector medial de las áreas 10, 11 y 12), se encuentra implicada en la respuesta emocional, en especial cuando la tarea a realizar conlleva contingencias aprendidas de refuerzo, debido a que esta región es una zona de convergencia de la estimulación exteroceptiva e interoceptiva (Aggleton, Burton y Passingham, 1980; Derryberry y Tucker, 1992). El estudio de la actividad neuronal de la corteza prefrontal ventromedial mediante técnicas de registro celular ha mostrado una mayor actividad ante estímulos visuales de contenido negativo o desagradable que ante otros contenidos afectivos (Kawasaki, Adolphs, Kaufman, Damasio, Damasio et al., 2001). Empleando técnicas electrofisiológicas, Carretié, Hinojosa, Mercado y Tapia (2005) han encontrado que en sujetos con miedo a las arañas se produce una mayor activación de la región prefrontal ventromedial cuando los estímulos no se perciben de forma consciente. Además, la reactividad de esta región es muy rápida, estando en torno a los $150 \mathrm{~ms}$. (Carretié et al., 2005; Carretié, Hinojosa, Albert y Mercado, 2006). Una función clave de esta región es realizar una reevaluación continua de las asociaciones emocionales, de modo que muestra ajustes rápidos a los cambios en el valor de las recompensas y en los estímulos asociados a ellas (Rolls, 1999). Dado que modula la actividad de un gran número de regiones, la corteza prefrontal ventromedial parece encontrarse implicada en mecanismos de regulación emocional. Se ha observado en monos, por ejemplo, que la lesión de esta región produce una alteración del control emocional inhibitorio (Dias, Robbins y Roberts, 1996). En línea con esta hipótesis, empleando técnicas de neuroimagen funcional algunos estudios han mostrado una disminución de actividad en esta región prefrontal en sujetos fóbicos expuestos a imágenes relacionadas con sus fobias, pero no ante otros contenidos desagradables, lo que indicaría una alteración en la regulación automática de las emociones provocadas por los estímulos fóbicos (Hermann, Schäfer, Walter, Stark, Vaitl y Schienle, 2007, 2009).

Una región estrechamente relacionada tanto con la corteza órbitofrontal como con la amígdala es la corteza cingulada anterior. Esta región se ha subdividido, a su vez, en una zona emocional y en una zona cognitiva (Morris y Dolan, 2004). La zona emocional se encuentra en su región ventral e implica las áreas de Brodmann 25, 32 y 33. Las células de esta región responden ante la significación y novedad de los estímulos, por lo que a esta región se le ha atribuido una función de tendencia o disposición a la acción (Damasio y Van Hoesen, 1984). Además de sus conexiones con la amígdala y la corteza prefrontal ventromedial, la corteza cingulada anterior también recibe proyecciones de la ínsula anterior (información relacionada con el estado interoceptivo) y, a su vez, envía proyecciones hacia núcleos vegetativos del tronco cerebral (Devinsky, Morrell y Vogt, 1995). Vogt, Finch y Olson (1992) han sugerido que la amígdala y la corteza cingulada anterior operan conjuntamente para generar emociones. Así, se ha encontrado que esta región se encuentra activa cuando se provocan síntomas en pacientes con trastornos de ansiedad (Rauch, Savage, Alpert, Miguel, Baer et al., 1995). En pacientes fóbicos, Pissiota y colaborado- 
res (2003) han encontrado una coactivación de la amígdala y de la corteza cingulada anterior (área 32) durante la modulación emocional del parpadeo reflejo de sobresalto en sujetos fóbicos. Los autores señalan que la activación del área 32 podría desempeñar un papel regulador en las experiencias emocionales de tipo defensivo, aumentando la atención a los estímulos amenazantes. De igual modo, Carretié y colaboradores (2009) han apuntado que la corteza cingulada anterior debe considerarse una estructura secundaria a la amígdala o a la corteza prefrontal ventromedial dentro de lo que denominan "cerebro negativo" (compuesto por la amígdala, corteza prefrontal ventromedial e ínsula anterior). Sin embargo un estudio reciente no ha encontrado una coactivación entre la amígdala y la corteza cingulada anterior en sujetos fóbicos expuestos a imágenes relacionadas con sus fobias (Ahs et al., 2009), sugiriendo los autores que la aparición del miedo fóbico se produciría debido a una alteración en el control que la corteza cingulada anterior ejercería sobre la amígdala. Otra función con la que se ha relacionado la corteza cingulada anterior es con la experiencia subjetiva de la ansiedad anticipadora, ya que se observa una relación entre la actividad de esta región, y la de la corteza prefrontal medial, y la ansiedad de los sujetos fóbicos durante la anticipación de imágenes relacionadas con sus fobias (Straube, Mentzel y Miltner, 2007).

\section{Ínsula}

La ínsula es una estructura cortical encargada de procesar sensaciones interoceptivas, relacionándose, particularmente su parte anterior, con el afecto, con situaciones negativas, con la anticipación de estímulos aversivos tales como el dolor y con la conciencia interoceptiva (Craig, 2002, 2009), aunque esta última función está siendo matizada en la actualidad (ver Khalsa, Rudrauf, Feinstein y Tranel, 2009). La ínsula recibe aferencias del tálamo, así como de otras regiones sensoriales. Esta convergencia de proyecciones hacen que esta región esté directamente implicada en la representación de los estados corporales internos conforme éstos se producen (Craig, 2009). Además, se encuentra relacionada tanto con la amígdala como con la corteza órbitofrontal. Mediante sus conexiones con la amígdala, el diencéfalo y el tronco del encéfalo, la ínsula controla diferentes funciones vegetativas (Goossens et al., 2007b). Como hemos visto anteriormente, la ínsula también envía proyecciones hacia la corteza cingulada anterior que, a su vez, envía eferencias hacia los núcleos vegetativos del tronco (Craig, 2002; Devinsky et al., 1995).

Al igual que ocurre con la amígdala, se ha observado un aumento de activación en la porción anterior de la ínsula durante el procesamiento de estímulos afectivos (Carr, Iacobini, Dubeau, Mazziota y Leinzi, 2003; Ishai, Pessoa, Bickle y Ungerleider, 2004; Pessoa y Padmala, 2005). Algunos autores señalan, además, que la actividad de esta región es muy importante en la representación cortical de los cambios vegetativos relacionados con la ansiedad (Davidson e Irwin, 1999). Como se ha visto anteriormente, la aparición del objeto fóbico produce un aumento de la actividad de la ínsula anterior (Etkin y Wager, 2007), por lo que algunos autores le atribuyen una función importante en el procesamiento de estímulos amenazantes (Carretié et al., 2009). Este hecho se ha comprobado en los estudios que han evaluado la activación cerebral mediante resonancia magnética funcional antes y después del tratamiento cognitivo-conductual de los sujetos fóbicos. Mientras que la exposición al objeto fóbico provoca un aumento de actividad de la ínsula anterior, el tratamiento atenúa dicha activación, así como la de otras regiones, que se asemeja a la que se observa en los sujetos no fóbicos (Goossens et al., 2007a; Straube et al., 2006a).

\section{Conclusión}

Las fobias específicas conllevan un conjunto de reacciones fisiológicas periféricas y centrales que acompañan a una fuerte percepción de temor o miedo y a conductas observables de evitación o huida. Estas respuestas ponen en evidencia que en el sujeto fóbico expuesto al objeto de su fobia se produce una activación del sistema de defensa que protege al organismo del estímulo amenazante. Esta reacción defensiva se manifiesta, a nivel periférico, en un aumento de la reactividad vegetativa y somática, produciendo, por ejemplo, cambios en el sistema cardiovascular (aumento de la frecuencia cardiaca e incremento de la vasoconstricción periférica) y en la respuesta electrodérmica (aumento de la magnitud de las respuestas de conductancia de la piel), así como la potenciación de los reflejos defensivos (adelanto del segundo componente acelerativo de la respuesta cardiaca de defensa y aumento de la magnitud del parpadeo reflejo defensivo). En humanos, se ha visto que esta reactividad aparece cuando el sujeto percibe los estímulos objeto de su fobia tanto de forma consciente como de forma no consciente. Esto conlleva plantear la existencia de mecanismos cerebrales de procesamiento preatencional, encargados de identificar y responder rápidamente a estímulos potencialmente amenazantes para la integridad del sujeto. Una de las principales estructuras cerebrales que responden de este modo es la amígdala cerebral. Esta estructura se encarga de identificar y preparar respuestas defensivas a estímulos amenazantes, con independencia de que éstos aparezcan dentro o fuera de la percepción consciente. Pero esta estructura no opera sola, sino conjuntamente con una amplia red de regiones cerebrales para identificar el estímulo amenazante y preparar las reacciones fisiológicas, conductuales y cognitivas que componen la respuesta defensiva provocada por dicho estímulo. Las estructuras cerebrales más importantes son la región órbitofrontal del lóbulo frontal (particularmente el sector ventromedial), la corteza cingulada anterior y la ínsula anterior. Estas regiones están interconectadas entre sí y realizan funciones complementarias en la detección de, y respuesta a, la estimulación amenazante. La investigación futura tendrá que matizar la función específica que desempeñan cada una de estas 
estructuras y su acción conjunta, así como su relación con los cambios vegetativos y motores periféricos que se aprecian en las fobias específicas.

\section{Referencias}

Aggleton, J.P. y Young, A.W. (2000). The enigma of the amygdala: on its contribution to human emotion. En R.D. Lane y L. Nadel (Eds.), Cognitive neuroscience of emotion (pp. 106-128). New York: Oxford University Press.

Aggleton, J.P., Burton, M.J. y Passingham, R.E. (1980). Cortical and subcortical afferents to the amygdala of the rhesus monkey. Brain Research, 190, 347-368.

Ahs, F., Pissiota, A., Michelgard, A., Frans, O., Furmark, T., Appel, L. y Frederikson, M. (2009). Disentangling the web of fear: amygdala reactivity and functional connectivity in spider and snake phobia. Psychiatry Research: Neuroimaging, 172, 103-108.

Barbas, H. (2000). Connections underlying the síntesis of cognition, memory, and emotion in primate prefrontal cortices. Brain Research Bulletin, 52, 319-330.

Ben-Ari, Y. (1981). Transmitters and modulators in the amygdaloid complex: a review. En Y. Ben-Ari (Ed.), The amygdaloid complex (pp. 163-174). New York: Elsevier.

Berntson, G.G. y Cacioppo, J.T. (2007). Integrative physiology: homeostasis, allostasis, and the orchestration of systemic physiology. En J.T. Cacioppo, L.G. Tassinary y G.G. Berntson (Eds.), Handbook of psychophysiology, 3a Ed. (pp. 433-452). Cambridge: Cambridge University Press

Berntson, G.G., Cacioppo, J.T. y Quigley, K.S. (1996). Cardiac psychophysiology and autonomic space in humans: empirical perspectives and conceptual implications. Psychological Bulletin, 114, 296-322.

Berntson, G.G., Cacioppo, J.T., Quigley, K.S. y Fabro, V.T. (1994). Autonomic space and psychophysiological response. Psychophysiology, 31, 44-61.

Berntson, G.G., Quigley, K.S. y Lozano, D. (2007). Cardiovascular psychophysiology. En J.T. Cacioppo, L.G. Tassinary y G.G. Berntson (Eds.), Handbook of psychophysiology, $3 a$ Ed. (pp. 182-210). Cambridge: Cambridge University Press.

Bradley, M.M. (2000). Emotion and motivation. En J.T. Cacioppo, L.G. Tassinary y G.G. Berntson (Eds.), Handbook of Psychophysiology, $2^{a}$ Ed. (pp. 602-642). Cambridge: Cambridge University Press.

Bradley, M.M. y Lang, P.J. (2000). Measuring emotion: behavior, feeling, and physiology. En R.D. Lane y L. Nadel (Eds.), Cognitive neuroscience of emotion (pp. 242-276). New York: Oxford University Press.

Bradley, M.M. y Lang, P.J. (2007). Emotion and motivation. En J.T. Cacioppo, L.G. Tassinary y G.G. Berntson (Eds.), Handbook of psychophysiology, $3 a$ Ed. (pp. 581-607). Cambridge: Cambridge University Press.
Brownley, K.A., Hurwitz, B.E. y Schneiderman, N. (2000). Cardiovascular psychophysiology. En J.T. Cacioppo, L.G. Tassinary y G.G. Berntson (Eds.), Handbook of Psychophysiology, $2^{a}$ Ed. (pp. 224-264). Cambridge: Cambridge University Press

Buodo, G., Peyk, P., Junghöher, M., Palomba, D. y Rockstroh, B. (2007). Electromagnetic indication of hypervigilant responses to emocional stimuli in blood-injection-injury fear. Neuroscience Letters, 424, 100-105.

Canli, T., Sivers, H., Whitfield, S.L., Gotlib, I.H. y Gabrieli, J.D.E. (2002). Amygdala response to happy faces as a function of extraversion. Science, 296, 2191.

Carlsson, K., Petersson, K.M., Lundqvist, D., Karlsson, A., Ingvar, M. y Öhman, A. (2004). Fear and the amygdala: manipulation of awareness generates differential cerebral responses to phobic and fear-relevant (but nonfeared) stimuli. Emotion, 4, 340-353.

Carr, L. Iacoboni, M., Dubeau, M.C., Mazziotta, J.C. y Lenzi, G.L. (2003). Neural mechanisms of empathy in humans: a relay from neural systems for imitation to limbic areas. Proceedings of the National Academy of Sciences, 100, 5497-5502.

Carretié, L., Albert, J., López-Martín, S. y Tapia, M. (2009). Negative brain: An integrative review on the neural processes activated by unpleasant stimuli. International Journal of Psychophysiology, 71, 57-63.

Carretié, L., Hinojosa, J.A., Albert, J. y Mercado, F. (2006). Neural response to sustained affective vidual stimulation using an indirect task. Experimental Brain Research, 174, 630-637.

Carretié, L., Hinojosa, J.A., Mercado, F. y Tapia, M. (2005). Cortical response to subjectively unconscious danger. $\mathrm{Neu}$ rolmage, 24, 615-623.

Cook, E. y Turpin, G. (1997). Differentiating orienting, startle and defense responses: The role of affect and its implications for psychopathology. En P.J. Lang, R.F. Simons y M. Balaban (Eds.), Attention and orienting: sensory and motivational processes (pp. 137-164). Mahwah, NJ: Lawrence Erlbaum.

Cook, E.W., Davis, T.L., Hawk, L.W., Spence, E.L. y Gautier, C.H. (1992). Fearfulness and startle potentiation during aversive visual stimuli. Psychophysiology, 29, 633-645.

Cook, E.W., Hawk, L.W., Davis, T.L. y Stevenson, V.E. (1991). Affective individual differences and startle reflex modulation. Journal of Abnormal Psychology, 100, 5-13.

Craig, A.D. (2002). How do you feel? Interoception: the sense of the physiological condition of the body. Nature Reviews Neuroscience, 3, 655-666.

Craig, A.D. (2009). How do you feel-now? The anterior insula and human awareness. Nature Reviews Neuroscience, 10, 59-70.

Critchley, H.D., Rotshtein, P., Nagai, Y., O’Doherty, J.O., Mathias, C.J. y Dolan, R.J. (2005). Activity in the human 
brain predicting differential heart rate responses to emotional facial expressions. NeuroImage, 24, 751-762.

Damasio, A. y Van Hoesen, G.W. (1984). Emotional disturbances associated with focal lesions of the limbic frontal lobe. En K.M. Heilman y P. Satz (Eds.), Neuropsychology of human emotion (pp. 85-110). New York: The Guilford Press.

Das, P., Kemp, A.H., Liddell, B.J., Brown, K.J., Olivieri, G., Peduto, A., Gordon, E. y Williams, L.M. (2005). Pathways for fear perception: modulation of amygdala activity by thalamo-cortical systems. NeuroImage, 26, 141-148.

Davidson, R.J. e Irwin, W. (1999). The functional neuroanatomy of emotion and affective style. Trends in Cognitive Sciences, 3, 11-21.

Davis, M. (1997). The neurophysiological basis of acoustic startle modulation: research on fear, motivation, and sensory gating. En P.J. Lang, R.F. Simons y M. Balaban (Eds.), Attention and orienting: sensory and motivational processes (pp. 69-96). Mahwah, New Jersey: Lawrence Erlbaum Associates.

Derryberry, D. y Tucker, D.M. (1992). Neural mechanisms of emotion. Journal of Consulting and Clinical Psychology, 60, 329-338.

Devinsky, O., Morrell, M.J. y Vogt, B.A. (1995). Contributions of anterior cingulated cortex to behaviour. Brain, 118, 279-306.

Dias, R., Robbins, T.W. y Roberts, A.C. (1996). Dissociation in prefrontal cortex of affective and attentional shifts. Nature, 380, 69-72.

Dilger, S., Straube, T., Mentzel, H.J., Fitzek, C., Reichenbach, J.R., Hecht, H. et al. (2003). Brain activation to phobiarelated pictures in spider phobic humans: an event-related functional magnetic resonance imaging study. Neuroscience Letters, 348, 29-32.

Emery, N.J. y Amaral, D.G. (2000). The role of the amygdala in primate social cognition, in Cognitive neuroscience of emotion. En R.D. Lane y L. Nadel (Eds.), Cognitive neuroscience of emotion (pp. 156-191). New York: Oxford University Press.

Etkin, A. y Wager, T.D. (2007). Functional neuroimaging of anxiety: a meta-analysis of emotional processing in PTSD, social anxiety disorder, and specific phobia. American Journal of Psychiatry, 164, 1476-1488.

Fanselow, M.S. (1994). Neural organization of the defense behaviour system responsible for fear. Psychonomic Bulletin and Review, 1, 429-438.

Fernández, M.C. y Vila, J. (1989). Sympathetic-parasympathetic mediation of the cardiac defense response in humans. Biological Psychology, 28, 123-133.

Fyer, A.J. (1998). Current approaches to etiology and pathophysiology of specific phobia. Biological Psychiatry, 44, 1295-1304.

Globisch, J., Hamm, A.O., Esteves, F. y Öhman, A. (1999). Fear appears fast: temporal course of startle reflex potentiation in animal fearful subjects. Psychophysiology, 36, 66-75.
Goossens, L., Schruers, K., Peeters, R., Griez, E. y Sunaert, S. (2007b). Visual presentation of phobic stimuli: amygdala activation via an extrageniculostriate pathway? Psychiatry Research: Neuroimaging, 155, 113-120.

Goossens, L., Sunaert, S., Peeters, R., Griez, E.J.L. y Schruers, K.R.J. (2007a). Amygdala hyperfunction in phobic fear normalizes after exposure. Biological Psychiatry, 62, 11191125 .

Graham, F. (1979). Distinguishing among orienting, defense, and startle reflexes. En H.D. Kimmel, E.H. Van Olst y J.F. Orlebeke (Eds.), The orienting reflex in humans (pp. 137167). Hillsdale, NJ: Lawrence Erlbaum.

Hamann, S.B., Ely, T.D., Hoffman, J.M. y Kilts, C.D. (2002). Ectasy and agony: activation of the human amygdala in positive and negative emotion. Psychological Science, 13, 135-141.

Hamm, A.O., Cuthbert, B.N., Globisch J. y Vaitl, D. (1997). Fear and the startle reflex: blink modulation and autonomic response patterns in animal and mutilation fearful subjects. Psychophysiology, 34, 97-107.

Hare, R.D. (1973). Orienting and defensive responses to visual stimuli. Psychophysiology, 10, 453-464.

Hare, R.D. y Blevings, G. (1975). Conditioned orienting and defensive responses. Psychophysiology, 12, 289297.

Hermann, A., Schäfer, A., Walter, B., Stark, R., Vaitl, D. y Schienle, A. (2009). Emotion regulation in spider phobia: role of the medial prefrontal cortex. Social Cognitive and Affective Neuroscience, 4, 257-267.

Hermann, A., Schäfer, A., Walter, B., Stark, R., Vaitl, D. y Schienle, A. (2007). Diminished medial prefrontal cortex activity in blood-injection injury phobia. Biological Psychology, 75, 124-130.

Ishai, A., Pessoa, L., Bickle, P.C. y Ungerleider, L.G. (2004). Repetition suppression of faces is modulated by emotion. Proceedings of the National Academy of Sciences, 101, 9827-9832.

Kawasaki, H., Adolphs, R., Kaufman, O., Damasio, H., Damasio, A., Granner, M. et al. (2001). Single-neuron responses to emotional visual stimuli recorded in human ventral prefrontal cortex. Nature Neuroscience, 4, 15-16.

Kemp, J.M. y Powell, T.P.S. (1970). The cortico-striate projections in the monkey. Brain, 93, 525-546.

Khalsa, S.S., Rudrauf, D., Feinstein, J.S. y Tranel, D. (2009). The pathways of interoceptive awareness. Nature Neuroscience, doi:10.1038/nn.2411

Klorman, R., Weissberg, R.P. y Wiesenfeld, A.R. (1977). Individual differences in fear and autonomic reactions to affective stimulation. Psychophysiology, 14, 45-51.

LaBar, K.S., LeDoux, J.E., Spencer, D.D. y Phelps, E.A. (1995). Impaired fear conditioning following unilateral temporal lobectomy in humans. The Journal of Neuroscience, 15, 6846-6855. 
Lane, R.D., Reiman, E.M., Bradley, M.M., Lang, P.J., Ahern, G.L., Davidson, R.J. y Schwartz, G.E. (1997). Neuroanatomical correlates of pleasant and unpleasant emotion. $\mathrm{Neu}$ ropsychologia, 35, 1437-1444.

Lang, P.J. (1995). The emotional probe. American Psychologist, 50, 372-385.

Lang, P.J., Bradley, M.M. y Cuthbert, B.N. (1990). Emotion, attention, and the startle reflex. Psychological Review, 97, 377-395.

Lang, P.J., Bradley, M.M., Cuthbert, B.N. (1997). Motivated attention: affect, activation, and action. En P.J. Lang, R.F. Simons y M. Balaban (Eds.), Attention and orienting: sensory and motivational processes (pp. 97-135). Mahwah, NJ: Lawrence Erlbaum.

Lang, P.J., Davis, M. y Öhman, A. (2000). Fear and anxiety: animal models and human cognitive psychophysiology. Journal of Affective Disorders, 61, 137-159.

Lang, P.J., Greenwald, M.K., Bradley, M.M. y Hamm, A.O. (1993). Looking at pictures: affective, facial, visceral, and behavior reactions. Psychophysiology, 30, 261-273.

Larson, C.L., Schaefer, H.S., Siegle, G.J., Jackson, C.A.B., Anderle, M.J. y Davidson, R.J. (2006). Fear is fast in phobic individuals: amygdala activation in response to fearrelevant stimuli. Biological Psychiatry, 60, 410-417.

LeDoux, J.E. (1987). Emotion. En F. Plum (Ed.), Handbook of physiology (Section 1). The nervous system. Vol. 5 (pp. 419460). Bethesda: American Physiological Society.

LeDoux, J.E. (1989). Cognitive-emotional interactions in the brain. Cognition and Emotion, 3, 267-289.

LeDoux, J.E. (1993). Emotional networks in the brain. En M. Lewis y J.M. Haviland (Eds.), Handbook of emotions (pp. 109-118). New York: Guilford Press.

LeDoux, J.E. (1998). Fear and the brain: where have we been, and where are we going? Biological Psychiatry, 44, 1229-1238.

LeDoux, J.E. (2000). Cognitive-emotional interactions: listen to the brain, in Cognitive neuroscience of emotion. En R.D. Lane y L. Nadel (Eds.), Cognitive neuroscience of emotion (pp. 129-155). New York: Oxford University Press.

LeDoux, J.E., Iwata, P., Cicchetti, P. y Reiss, D.J. (1988). Different projections of the central amygdaloid nucleus mediate autonomic and behavioral correlates of conditioned fear. Journal of Neuroscience, 8, 2517-2529.

Masterson, F.A. y Crawford, M (1982). The defense motivation system: a theory of avoidance behaviour. Behavioral and Brain Sciences, 5, 661-696.

Morris, J. y Dolan RJ. (2004). Functional neuroanatomy of human emotion. En R.S.J. Frackowiak, K.J. Friston, C.D. Frith, R.J. Dolan, C.J. Price, S. Zeki, J. Ashburner y W. Penny (Eds.), Human brain function. $2^{a}$ Ed. (pp. 365-396). Londres: Elsevier

Morris, J.S., Frith, C.D., Perrett, D.I., Rowland, D., Young, A.W., Calder, A.J. et al. (1996). A differential neural response in the human amygdala to fearful and happy facial expresions. Nature, 383, 812-815.

Mühlberger, A., Wiedemann, G., Hermann, M.J. y Pauli, P. (2006). Phylo- and ontogenetic fears and the expectation of danger: differences between spider- and flight-phobic subjects in cognitive and physiological responses to disorder-specific stimuli. Journal of Abnormal Psychology, 115, 580-589.

Murray, E.A. (2007). The amygdala, reward and emotion. Trends in Cognitive Sciences, 11, 489-497.

Nauta, W.J.H. (1964). Some efferent connections of the prefrontal cortex in the monkey. En J.M. Warren y K. Akert (Eds.), The frontal granular cortex and behavior. New York: McGraw Hill.

Öhman, A. y Soares, J.F. (1994). "Unconscious anxiety": Phobic responses to masked stimuli. Journal of Abnormal Psychology, 103, 231-240.

Papillo, J.F. y Shapiro, D. (1990). The cardiovascular system. En J.T. Cacioppo y L.G. Tassinary (Eds.), Principles of psychophysiology: physical, social, and inferential elements (pp. 456-512). New York: Cambridge University Press.

Paradiso, S., Johnson, D.L., Andreasen, N.C., O’Leary, D.S., Watkins, G.L., Ponto, L.L. et al. (1999). Cerebral blood flow changes associated with attribution of emotional valence to pleasant, unpleasant, and neutral visual stimuli in a PET study of normal subjects. American Journal of Psychiatry, 156, 1618-1629.

Pessoa, L. y Padmala, S. (2005). Quantitative prediction of perceptual decisions during near-thresholds fear detection. Proceedings of the National Academy of Sciences, 102, 5612-5617.

Phan, K.L., Wager, T., Taylor, S.F. y Liberzon, I. (2002). Functional neuroanatomy of emotion: a meta-analysis of emotion activation studies in PET and fMRI. NeuroImage, 16, 331-348.

Pissiota, A., Frans, Ö., Michelgard, A., Appel, L., Langström, B., Flaten, M.A. y Frederikson, M. (2003). Amygdala and anterior cingulate cortex activation during affective startle modulation: a PET study of fear. European Journal of Neuroscience, $18,1325-1331$.

Quigley, K.S. y Berntson, G.G. (1990). Autonomic origins of cardiac responses to nonsignal stimuli in the rat. Behavioral Neuroscience, 104, 751-762.

Ramírez, I., Sánchez, M.B., Fernández, M.C., Lipp, O.V. y Vila, J. (2005). Differentiation between protective reflexes: cardiac defense and startle. Psychophysiology, 42, 732-739.

Rauch, S.L., Savage, C.R., Alpert, N.M., Miguel, E.C., Baer, L. et al. (1995). A positron emission tomographic study of simple phobic symptom provocation. Archives of General Psychiatry, 52, 20-28.

Reyes del Paso, G., Godoy, J. y Vila, J. (1993). Respiratory sinus arrhythmia as an indes of parasympathetic cardiac control during the cardiac defense response. Biological Psychology, 35, 17-35. 
Reyes del Paso, G., Vila, J. y García, A. (1994). Physiological significance of the defense response to intense auditory stimulation: a pharmacological blockade study. International Journal of Psychophysiology, 17, 181-187.

Rolls, E.T. (1986). Neural systems involved in emotion in primates. En R. Plutchik y H. Kellerman (Eds.), Emotion: theory, research, and experience. Vol. 3 (pp. 125-143). New York: Academic Press.

Rolls, E.T. (1999). The brain and emotion. Oxford: Oxford University Press.

Ruiz-Padial, E. y Vila, J. (2007). Fearful and sexual pictures not consciously seen modulate the startle reflex in human beings. Biological Psychiatry, 61, 996-1001.

Ruiz-Padial, E., Mata, J.L., Rodríguez, S., Fernández, M.C. y Vila, J. (2005). Non-conscious modulation of cardiac defense by masked phobic pictures. International Journal of Psychophysiology, 56, 271-281.

Sabatinelli, D., Bradley, M.M., Fitzsimmons, J.R. y Lang, P.J. (2005). Parallel amygdala and inferotemporal activation reflect emotional intensity and fear relevance. NeuroImage, 24, 1265-1270.

Sánchez, M.B., Ruiz-Padial, E., Pérez, N., Fernández, M.C., Cobos, P. y Vila, J. (2002). Modulación emocional de los reflejos defensivos mediante visualización de imágenes afectivas. Psicothema, 14, 702-707.

Sánchez-Navarro, J.P. y Martínez-Selva, J.M. (2009). The cardiac response of a threatened brain. En L. Sher (Ed.), Psychophysiological factors and cardiovascular disorders: the role of stress and psychosocial influences (pp. 113-137). New York: Nova Science Publishers.

Sánchez-Navarro, J.P., Martínez-Selva, J.M. y Román, F. (2006). Uncovering the relationship between defence and orienting in emotion: cardiac reactivity to unpleasant pictures. International Journal of Psychophysiology, 61, 34-46.

Sánchez-Navarro, J.P., Martínez-Selva, J.M., Torrente, G. y Román, F. (2008). Psychophysiological, behavioral, and cognitive indices of the emotional response: a factoranalytic study. The Spanish Journal of Psychology, 11, 16-25.

Sánchez-Navarro, J.P. y Román, F. (2004). Amígdala, corteza prefrontal y especialización hemisférica en la experiencia y expresión emocional. Anales de Psicología, 20, 223-240.

Sarlo, M., Buodo, G., Munafló, M., Stegagno, L. y Palomba, D. (2008). Cardiovascular dynamics in blood phobia: evidence for a key role of sympathetic activity in vulnerability to syncope. Psychophysiology, 45, 1038-1045.

Sarlo, M., Palomba, D., Angrilli, A. y Stegagno, L. (2002). Blood phobia and spider phobia: two specific phobias with different autonomic cardiac modulations. Biological Psychology, 60, 91-108.
Sokolov, E.N. (1963). Perception and the conditioned reflex. Oxford: Pergamon Press.

Straube, T., Glauer, M., Dilger, S., Mentzel, H.J. y Miltner, W.H. (2006a). Effects of cognitive-behavioral therapy on brain activation in specific phobia. NeuroImage, 29, 125135.

Straube, T., Mentzel, H.-J. y Miltner, W.H.R. (2007). Waiting for spiders: brain activation during anticipatory anxiety in spider phobics. NeuroImage, 37, 1427-1436.

Straube, T., Mentzel, H.-J., Miltner, W.H.R. (2006b). Neural mechanisms of automatic and direct processing of phobogenic stimuli in specific phobia. Biological Psychiatry, 59, 162-170.

Tabert, M.H., Borod, J.C., Tang, C.Y., Lange, G., Wei, T.C., Johnson, R. et al. (2001). Differential amygdala activation during emotional decision and recognition memory tasks using unpleasant words: An fMRI study. Neuropsychologia, 39, 556-573.

Van Hoesen, G.W., Pandya, D.N. y Butters, N. (1975). Some connections of the enthorhinal (Area 28) and perirhinal (Area 35) cortices in the monkey. III. Efferent connections. Brain Research, 95, 25-38.

Vila, J., Fernández, M.C., Pegalajar, J., Vera, M.N., Robles, H., Pérez, N. et al. (2003). A new look at cardiac defense: attention or emotion? The Spanish Journal of Psychology, 6, 60-78.

Vila, J., Guerra, P., Muñoz, M.A., Vico, C., Viedma-Del Jesús, M.I., Delgado, L.C. et al. (2007). Cardiac defense: from attention to action. International Journal of Psychophysiology, 66, 169-182.

Vogt, B.A., Finch, D.M. y Olson, C.R. (1992). Functional heterogeneity in cingulate cortex: the anterior executive and posterior evaluative regions. Cerebral Cortex, 2, 435-443.

Wendt, J., Lotze, M., Weike, A.I., Hosten, N. y Hamm, A.O. (2008). Brain activation and defensive response mobilization during sustained exposure to phobia-related and other affective pictures in spider phobia. Psychophysiology, 45, 205-215.

Fecha de recepción: 16 de noviembre de 2009 Fecha de aceptación: 9 de diciembre de 2009 